\title{
Fluid description of kinetic modes
}

\author{
Y. Y. Lau \\ Intense Energy Beam Interaction Laboratory and Department of Nuclear Engineering, \\ University of Michigan, Ann Arbor, Michigan 48109-2104
}

(Received 7 March 1994; accepted 16 May 1994)

\begin{abstract}
The classical kinetic modes, such as the Bernstein mode, the loss cone modes, and the Harris dispersion relation are reconstructed from a fluid-like analysis. The analysis begins with a delta function in the equilibrium distribution. By simply calculating the displacement of a single electron exposed to a small signal electric field, the charge perturbation density, and the dispersion relation, immediately follow. The effect of a velocity distribution enters only through a trivial integration of the dispersion relation thus obtained for the monoenergetic plasma. The entire analysis is done in the configuration space. Thus, without explicitly performing the customary integration over the unperturbed orbits in phase space, finite Larmor radius effects to all order have been retained. Possible extensions to nonuniform plasma and to nonuniform magnetic fields are indicated.
\end{abstract}

\section{INTRODUCTION}

Microinstabilities, kinetic modes, and velocity-space instabilities have occupied a central position in plasma physics for almost 40 years. ${ }^{1}$ Although they carry slightly different meanings, these three terms have often been used interchangeably, whenever their description requires a detailed consideration of the charged particle dynamics in the phase space, $\left(x, y, z, v_{x}, v_{y}, v_{z}\right)$. Their importance to anomalous transport has been recognized from the outset.

Analyses of kinetic modes typically begin with a specification of the equilibrium distribution function, to be followed by an integration of the linearized Vlasov equation over the unperturbed orbits in the six-dimensional phase space, when an electromagnetic perturbation is imposed. Prime examples ${ }^{1}$ of such modes are the drift modes and the loss cone instabilities. The stable Bernstein modes are also considered as kinetic modes. They are not amendable to a fluid treatment according to conventional wisdom, by which the fluid approach is judged to be capable of only treating a continuum in the three-dimensional configuration space, $(x, y, z)$.

In this paper, we reconstruct several classical kinetic modes from what amounts to be essentially a fluid dynamical treatment. Virtually all of the analyses are performed in the configuration space, for a monoenergetic plasma. The effect of a general velocity distribution is included at the end by a trivial superposition. In fact, the entire calculation involves little more than an evaluation of the perturbed displacement of a typical electron (or ion) when a small signal electromagnetic field is present. Direct integration of the linearized Vlasov equation is avoided altogether. Specifically, we shall use such a procedure to recover the Harris dispersion relation ${ }^{2}$ from which the Dory-Guest-Harris loss cone modes ${ }^{3}$ and the Bernstein modes ${ }^{4}$ may be obtained as a special case. Note that, in so doing, finite Larmor radius effects have been retained to all order.

There are several reasons why velocity space instabili- ties can be reconstructed by an equivalent, but conceptually much simpler analysis in the configuration space. First, the linearized Vlasov equation is linear in the equilibrium distribution function $f_{0}$ [Fig. 1(a)]. Thus we may envision this equilibrium distribution function to be a superposition of delta functions. The total response, in the linearized charge density or in the linearized current density, is then a straightforward superposition of that due to a delta function distribution [Fig. 1(b)]. For a delta function distribution, the medium is infinitely cold, and we can unambiguously label an electron by its condition at time $t=0$. Let $\left(x_{0}, y_{0}, z_{0}\right)$ be its unperturbed coordinates at time $t$. The perturbation density may then be calculated in these independent variables $\left(x_{0}, y_{0}, z_{0}, t\right)$, which are also known as the "polarization variables" in the microwave tube literature. ${ }^{5}$ The polarization variables follow fluid motion like the Lagrangian variables, but are equivalent to (and convenient as) the more familiar Eulerian variables $(x, y, z, t)$ through a straightforward transformation. ${ }^{5}$ This observation prompted the analysis in the configuration space. Finally, since we are considering collective modes in a plasma, all electrons and ions experience the same electromagnetic field that is expressed in the Eulerian variables $(x, y, z, t)$. Thus, calculation of the displacement, $x_{1}$, of one electron suffices. The small signal density immediately follows. [See Eq. (3) below.]

The arguments given in the preceding paragraph could perhaps have been anticipated by referring to the familiar plasma dispersion relation, given by Eq. (10) below. That equation exhibits Landau damping, the arch property of kinetic effects. It may be constructed by a simple superposition [Eq. (9)] of the space charge wave ${ }^{1}$ that is obtained on a monocnergetic elcctron strcam [Eq. (8)].

In this paper, we shall restrict our attention only to the electrostatic waves in a uniform, nonrelativistic, magnetized plasma. Individually studied are the modes propagating parallel (Sec. III) and perpendicular (Sec. IV) to the external 
(a)

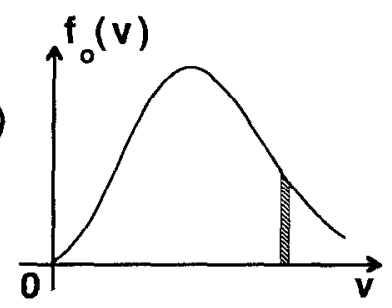

(b)

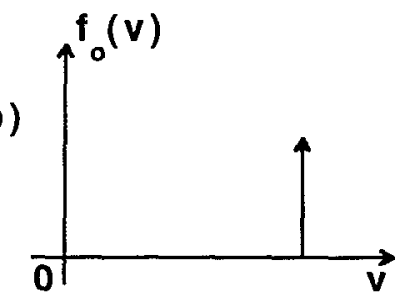

FIG. 1. (a) A general equilibrium distribution function and (b) its decomposition into delta functions. Here, $v$ represents the velocity component either parallel to, or perpendicular to, the external magnetic field.

magnetic field. They are combined to yield the Harris dispersion relation (Sec. V).

Although no new results are reported in this paper, some insight into velocity space instability is offered. For example, the dispersion relation derived in an alternative form allows a closer connection between the Dory-Guest-Harris instability and the space charge waves in a magnetized plasma. The techniques developed here may easily be extended to electromagnetic modes. Perhaps more importantly, they may also be applied to an equilibrium that contains spatial gradients in density, in tempcrature, and in magnetic field. Such an analysis would involve mainly a calculation of the electron (or ion) displacement perturbation, and, as a result, would reduce the substantial entanglement in the $(\mathbf{x}, \mathbf{v})$ variables that always accompanies the traditional Vlasov formulation. Some of these issues, and the possible generalizations of the present studies, will be discussed in the last section.

\section{THE MODEL}

To fix ideas, consider a uniform plasma in which the ions are immobile and form a neutralizing background. This plasma is subject to a uniform external magnetic field $\mathbf{B}_{0}=\hat{z} B_{0}$. Consider a small signal electrostatic field,

$\mathbf{E}_{1}=\mathbf{E}_{1 o} \exp (i \omega t-i \mathbf{k} \cdot \mathbf{x})=i \mathbf{k} \phi_{1} \exp (i \omega t-i \mathbf{k} \cdot \mathbf{x})$,

on this equilibrium. We shall derive the density response to this electric field, and hence the dispersion relation, first for a monoenergetic electron plasma whose equilibrium velocity is $\left(v_{\perp}, v_{z}\right)$ where $v_{\perp}$ is the velocity perpendicular to the external magnetic field and $v_{z}$ is that parallel to the external magnetic field. The total density response is simply the sum over the equilibrium velocity distribution $f_{0}\left(v_{1}, v_{z}\right)$, and this is done only at the very last step. It involves no more than inserting the integral $\int d^{3} v f_{0}(v)$ into the dispersion relation obtaincd for the monoencrgetic plasma.

The fluid analysis is most conveniently done using the polarization variable, $\left(\mathbf{x}_{0}, t\right)$, defined as follows. Let $\mathbf{x}_{0}$ be the unperturbed position of an electron fluid element of the mo- noenergetic plasma, and $n_{0}$ be the number density attached to this element in equilibrium. Let $x_{1}=x_{1}\left(x_{0}, t\right)$ be the displacement of this element from its equilibrium position in the presence of an electrostatic perturbation, Eq. (1). Thus the instantaneous position of this element is

$$
\mathbf{x}=\mathbf{x}_{0}+\mathbf{x}_{1}\left(\mathbf{x}_{0}, t\right) .
$$

In terms of $x_{1}$, the number density perturbation $n_{1}$ is given by $^{5}$

$$
n_{1}\left(\mathbf{x}_{0}, t\right)=-\nabla \cdot\left[n_{0} \mathbf{x}_{1}\left(\mathbf{x}_{0}, t\right)\right]
$$

We remark that Eq. (3) is valid even when the equilibrium density $n_{0}$ is a function of $\mathbf{x}$. The dispersion relation is obtained by requiring that the Poisson equation, $\nabla \cdot \mathbf{E}_{1}=e n_{1} / \epsilon_{0}$, be satisfied. For a uniform plasma, it reads

$$
-i \mathbf{k} \cdot \mathbf{E}_{1}=k^{2} \phi_{1}=\left(e n_{0} / \epsilon_{0}\right) i \mathbf{k} \cdot \mathbf{x}_{1}
$$

upon using Eq. (3). Here, $k^{2}=\mathbf{k} \cdot \mathbf{k}$. When operating on linearized quantities, such as in Eqs. (3) and (4), all spatial derivatives in the Eulerian variables, $\partial / \partial x$ for instance, may be replaced by $\partial / \partial x_{0}$ in the polarization variables, the error is second order in $\mathbf{x}_{1}$.

In the next section, we shall describe the density response in these variables. To gain familiarity, we shall first revisit the simple problem of a cold, nonmagnetized, plasma with only a monoenergetic velocity $v_{z}$ in the equilibrium state. The familiar plasma dispersion relation, which encompasses Landau damping, is obtained by summing the density response over the equilibrium distribution function. These simple results may readily be extended to the slightly more complicated cases involving a nonzero equilibrium magnetic field.

\section{PLASMA OSCILLATION AND LANDAU DAMPING}

In this section, we set $\mathbf{B}_{0}=0$. Consider first a monoenergetic electron stream whose equilibrium velocity is $\mathbf{v}_{0}=\hat{z} v_{z}$. Then, the equilibrium coordinates $z_{0}$ of an electron fluid element is given by

$$
z_{0}=v_{z} t+p
$$

where $p$ is a constant designating the element at time $t=0$. With $\mathbf{k}=\hat{z} k_{z}$ and $\mathbf{E}_{1 o}=\hat{z} E_{1 o}$, Eq. (1) describes the small signal electric field acting on the displacement $z_{1}$ :

$$
\ddot{z}_{1}=\frac{e}{m} E_{10} e^{i \omega t-i k_{z}\left(v_{z} t+p\right)},
$$

where the dot $(\cdot)$ denotes the time derivative, and we have replaced $\mathbf{x}$ by $\mathbf{x}_{0}=\hat{z} z_{0}=\hat{z}\left(v_{z} t+p\right)$ in Eq. (1) since $E_{10}$ is already a small signal quantity. The steady state solution to Eq. (6) is

$$
z_{1}=-\frac{e}{m} \frac{E_{10} e^{i \omega t-i k_{z} z_{\curvearrowleft}}}{\left(\omega-k_{z} v_{z}\right)^{2}}
$$

where $z_{0}$ is given by Eq. (5). Substitution of Eq. (7) into Eq. (4) yields the familiar dispersion relation for the space charge waves on a monoenergetic electron plasma:

$$
\epsilon(\omega)=1-\frac{\omega_{p}^{2}}{\left(\omega-k_{z} v_{z}\right)^{2}}=0 .
$$


In Eq. (8), $\omega_{p}$ denotes the electron plasma frequency.

We may now superimpose the density response for a general equilibrium velocity distribution $f_{0}\left(v_{z}\right)$, normalized so that $\int d v_{z} f_{0}\left(v_{z}\right)=1$. Equation (8) is modified to read

$$
\epsilon(\omega)=1-\omega_{p}^{2} \int d v_{z} \frac{f_{0}\left(v_{z}\right)}{\left(\omega-k_{z} v_{z}\right)^{2}}=0 .
$$

The alternative form to Eq. (9) is obtained from integration by parts:

$$
\epsilon(\omega)=1-\frac{\omega_{p}^{2}}{k_{z}^{2}} \int d v_{z} \frac{\partial f_{0}\left(v_{z}\right) / \partial v_{z}}{v_{z}-\omega / k_{z}}=0 .
$$

The dispersion relation (9), or (10), is analytic for $\operatorname{Im}(\omega)<0$ by the causality condition. ${ }^{1}$ Landau damping appears when $\epsilon(\omega)$ is analytically continued to $\operatorname{Im}(\omega)=0$.

Since Landau damping may be described by Eq. (9) which is directly obtainable from the cold fluid theory, Eq. (8), it is then hardly surprising that all linear instabilities involving a general velocity distribution [Fig. 1(a)] may be constructed from a similar fluid-like theory that is being developed for a monoenergetic plasma [Fig. 1(b)].

\section{NONZERO EXTERNAL MAGNETIC FIELD $\left(B_{0} \neq 0\right)$}

To reconstruct the dispersion relations for the DoryGuest-Harris instability and for the Bernstein mode, we shall first revisit the upper hybrid mode, which describes the fundamental space charge wave in a plasma that is immersed in a uniform magnetic field $\hat{z} B_{0}$. As we shall see, the upper hybrid mode is closely related to the Bernstein mode and to the Dory-Guest-Harris loss cone instabilities. All of these modes can be analyzed under the simplifying assumptions $k_{z}=0$ and $v_{z}=0$ for all electrons in the equilibrium state. These assumptions are taken in this section; they will be removed in the next section.

\section{A. Upper hybrid mode}

The upper hybrid mode may readily be constructed by considering a cold electron plasma in a uniform magnetic field. For an electron fluid element that is at rest in equilibrium, $\mathbf{x}_{0}=$ constant. When we assume $\mathbf{k}=\hat{x} k_{x}$, the linearized force law for this fluid element reads:

$$
\begin{aligned}
& \ddot{x}_{1}=(e / m)\left(E_{1 x}+\dot{y}_{1} B_{0}\right), \\
& \ddot{y}_{1}=-(e / m) \dot{x}_{1} B_{0},
\end{aligned}
$$

which may be combined to yield:

$$
\ddot{x}_{1}+\Omega^{2} x_{1}=(e / m) E_{1 x} e^{i \omega t-i k_{x} x_{0}},
$$

where $\Omega=e B_{0} / m$ is the electron cyclotron frequency. The solution to Eq. (11) is simply

$$
x_{1}=\frac{e E_{1 x} e^{i \omega t-i k_{x} x_{0}}}{m\left(-\omega^{2}+\Omega^{2}\right)},
$$

which, logether wilh Eq. (4), yields

$$
\epsilon(\omega)=1-\frac{\omega_{p}^{2}}{\omega^{2}-\Omega^{2}}=0
$$

or,

$$
\omega^{2}=\Omega^{2}+\omega_{p}^{2}
$$

from which the upper hybrid frequency $\omega_{\mathrm{UH}}=\sqrt{\Omega^{2}+\omega_{p}^{2}}$ is obtained. Note that the dispersion relation (13b) follows naturally from the force law (11), which represents the restoring force on $x_{1}$ due to the axial magnetic field $(\Omega)$ and to the tendency against charge separation $\left(\omega_{p}\right)$.

\section{B. The Dory-Guest-Harris Instability}

The Dory-Guest-Harris instability ${ }^{3}$ is traditionally considered as the prime example of phase space instability due to anisotropy in the distribution function in phase space. The extreme case of anisotropy is when the equilibrium distribution function assumes a Delta function distribution in both $v_{z}$ and in $v_{\perp}$ [Fig. 1(b)]. This monoenergetic electron plasma may be treated in the polarization variables, as follows.

Again, with $k_{z}=0$ and $v_{z}=0$, Eq. (11) still applies to this monoenergetic plasma medium, except that $x_{0}$ in that equation accounts for the cyclotron motion in the unperturbed state:

$$
x_{0}=x_{g}+\frac{v_{1}}{\Omega} \sin (\Omega t+\varphi),
$$

where the constants $x_{g}$ and $\varphi$ are used to label the electronic element under consideration. Upon substituting Eq. (14) into (11), and using the Bessel function identity,

$$
e^{i b \sin \theta}=\sum_{n=-\infty}^{\infty} J_{n}(b) e^{i n \theta},
$$

in the factor $e^{-i k_{x} x_{0}}$, we rewrite (11) as

$\ddot{x}_{1}+\Omega^{2} x_{1}=\frac{e}{m} E_{1 x} e^{i \omega t-i k_{x} x_{g}} \sum_{n=-\infty}^{\infty} J_{n}\left(\frac{k_{x} v_{1}}{\Omega}\right) e^{-i m(\Omega t+\varphi)}$.

Equation (16) is that for a vibrating spring of natural frequency $\Omega$. This spring is being acted upon by an external force, represented by the right hand member of (16). The latter consists of infinitely many harmonic frequencies, $\omega-n \Omega$. The steady state solution to (16) is easily recognized to be:

$x_{1}=\frac{e}{m} E_{1 x} e^{i \omega t-i k_{x} x_{g}} \sum_{n=-\infty}^{\infty} \frac{J_{n}\left(k_{x} v_{1} / \Omega\right) e^{-i n(\Omega t+\varphi)}}{-(\omega-n \Omega)^{2}+\Omega^{2}}$.

This solution is also presented in Chen's book (Ref. 1, p. 275) when kinetic effects are discussed. The Poisson equation, (4), then reads:

$$
-i k_{x} E_{1 x} e^{i \omega t-i k_{x} x_{0}}=\left(e n_{0} / \epsilon_{0}\right) i k_{x} x_{1},
$$

in which $x_{1}$ is given by (17).

The dispersion relationship may be obtained by multiplying both sides of (18) by $e^{-i \omega t+i k_{x} x_{0}}$, with $x_{0}$ being given by (14), and using the Bessel identity (15) in $e^{i k_{x} x_{0}}$, and finally averaging over a cyclotron period. ${ }^{6}$ This leads to the dispersion relation 


$$
\epsilon(\omega)=1-\omega_{p}^{2} \sum_{n=-\infty}^{\infty} \frac{J_{n}^{2}\left(k_{x} v_{\perp} / \Omega\right)}{(\omega-n \Omega)^{2}-\Omega^{2}}=0,
$$

which may be shown (Appendix A) to be identical to the dispersion relation for the Dory-Guest-Harris instability for a delta function distribution in $v_{\perp}$ (Ref. 3):

$$
\epsilon(\omega)=1-\frac{\omega_{p}^{2}}{\Omega^{2}} \sum_{n=-\infty}^{\infty}\left(\frac{n \Omega}{\omega-n \Omega}\right) \frac{1}{b} \frac{d}{d b} J_{n}^{2}(b)=0 .
$$

In Eq. (20), $b=k_{x} v_{1} / \Omega$.

Equation (19) has a natural interpretation near the resonance condition $(\omega-n \Omega)^{2} \approx \Omega^{2}$, in the case of a tenuous plasma. It becomes

$$
(\omega-n \Omega)^{2} \approx \Omega^{2}+\omega_{p}^{2} J_{n}^{2}\left(k_{x} v_{\perp} / \Omega\right),
$$

which may be considered as the upper hybrid mode [Eq. (13b)] at the $n$th cyclotron harmonic. The geometrical factor $J_{n}^{2}\left(k_{x} v_{\perp} / \Omega\right)$ in Eq. (21) is the "reduction factor" which, in one form or other, always accompanies space charge waves. ${ }^{7}$ Equation (21) also invites a comparison with the force law (16). In so doing, we provide a derivation, from first principle, of the dispersion relation (19); the physics it displays appears to be a lot more transparent than its equivalent form, Eq. (20).

\section{Bernstein mode}

Bernstein mode ${ }^{4}$ in its simplest form, is an electrostatic wave propagating across a magnetized plasma which has a Maxwellian distribution function. We may construct the dispersion relation for the Bernstein mode simply by summing the density response due to various monoenergetic "electron fluids" that make up the Maxwellian distribution. Thus, Eq. (19) immediately yields the dispersion relation for the Bernstein mode propagating across the external magnetic field $\left(k_{z}=0\right)$ :

$\epsilon(\omega)=1-\omega_{p}^{2} \sum_{n=-\infty}^{\infty} \frac{\int d^{3} \mathbf{v} f_{0}(\mathbf{v}) J_{n}^{2}\left(k_{x} v_{\perp} / \Omega\right)}{(\omega-n \Omega)^{2}-\Omega^{2}}=0$,

when $f_{0}$ is a Maxwellian distribution function in equilibrium.

We shall postpone, to the next section, proving that (22) in fact describes the Bernstein mode. There, we construct the general Harris dispersion relation. All of the modes considered thus far in this paper may be considered as a special case of the Harris dispersion relation.

\section{HARRIS DISPERSION RELATION}

The Harris dispersion relation ${ }^{2}$ describes the general electrostatic wave in a uniform plasma subject to an external magnetic field $\hat{z} B_{0}$. The mode is represented by Eq. (1) with $\mathbf{k}=\hat{x} k_{x}+\hat{z} k_{z}$ and the equilibrium distribution function $f_{0}(v)=f_{0}\left(v_{1}, v_{2}\right)$ is an arbitrary function of the constants of motion, $v_{z}$ and $v_{\perp}$, in the unperturbed state. The Harris dispersion relation reads ${ }^{2,3}$

$$
\begin{aligned}
k_{x}^{2}+k_{z}^{2}= & \omega_{p}^{2} \sum_{n=-\infty}^{\infty} \int d^{3} \mathbf{v}\left\{J_{n}^{2}\left(\frac{k_{x} v_{\perp}}{\Omega}\right)\right. \\
& \left.\times\left[\frac{n \Omega}{v_{\perp}} \frac{\partial f_{0}}{\partial v_{\perp}}+k_{z} \frac{\partial f_{0}}{\partial v_{z}}\right]\right\} / k_{z} v_{z}+n \Omega-\omega,
\end{aligned}
$$

which is traditionally derived by an integration over the unperturbed orbits in the six-dimensional phase space, $(\mathbf{x}, \mathbf{v})$. Here, we reconstruct this dispersion relation from a fluid description. We basically combine the longitudinal propagation and the transverse propagation results that have already been described in Secs. III and IV, with only minor modifications. The effect of velocity distribution is inserted in the last step.

We may still use Eqs. (5) and (14) to designate the unperturbed coordinates, $x_{0}$ and $z_{0}$, of a monoenergetic fluid element. When subjected to a small signal electrostatic field, Eq. (1), the displacement from the unperturbed position is governed by [cf., Eqs. (11) and (6)]:

$$
\begin{aligned}
& \ddot{x}_{1}+\Omega^{2} x_{1}=(e / m)\left(i k_{x} \phi_{1}\right) e^{i \omega t-i k_{x} x_{0}-i k_{z} z_{0}}, \\
& \ddot{z}_{1}=(e / m)\left(i k_{z} \phi_{1}\right) e^{i \omega t-i k_{x} x_{0}-i k_{z} z_{0}} .
\end{aligned}
$$

These two equations may be solved for $x_{1}$ and $z_{1}$ by inserting Eqs. (5) and (14) into the right-hand members of (24), (25), and then applying the Bessel function identity (15). As before, this procedure yields the steady state solutions

$$
\begin{aligned}
x_{1}= & \frac{e}{m}\left(i k_{x} \phi_{1}\right) e^{i \omega t-i k_{x} x_{g}} \\
& \times \sum_{n=-\infty}^{\infty} \frac{J_{n}\left(k_{x} v_{\perp} / \Omega\right) e^{-i n(\Omega t+\varphi)-i k_{z}\left(v_{z} t+p\right)}}{-\left(\omega-n \Omega-k_{z} v_{z}\right)^{2}+\Omega^{2}}, \\
z_{1}= & \frac{e}{m}\left(i k_{z} \phi_{1}\right) e^{i \omega t-i k_{x} x_{g}} \\
& \times \sum_{n=-\infty}^{\infty} \frac{J_{n}\left(k_{x} v_{\perp} / \Omega\right) e^{-i n(\Omega t+\varphi)-t k_{z}\left(v_{z} t+p\right)}}{-\left(\omega-n \Omega-k_{z} v_{z}\right)^{2}},
\end{aligned}
$$

which may readily be compared with the solution (17). Analogous to Eq. (18), the Poisson equation (4) now reads

$$
\left(k_{x}^{2}+k_{z}^{2}\right) \phi_{1} e^{i \omega t-i k_{x} x_{0}-i k_{z} z_{0}}=\left(e n_{0} / \epsilon_{0}\right)\left(i k_{x} x_{1}+i k_{z} z_{1}\right) \text {. }
$$

Similarly, the dispersion relationship may be obtained by ${ }^{6}$ (i) substituting (26) and (27) into the right-hand member of (28), (ii) multiplying both sides of (28) by $e^{-i \omega t+i k_{x} x_{0}+i k_{z} z_{0}}$, (iii) expressing $x_{0}$ by (14) and $z_{0}$ by (5) and using the Bessel identity (15) in $e^{i k_{x} x_{0}}$, and finally, (iv) averaging over a cyclotron period. This leads to the dispersion relation

$$
\begin{aligned}
k_{x}^{2}+k_{z}^{2}= & \omega_{p}^{2} \sum_{n=-\infty}^{\infty} J_{n}^{2}\left(\frac{k_{x} v_{\perp}}{\Omega}\right)\left[\frac{k_{x}^{2}}{\left(\omega-n \Omega-k_{z} v_{z}\right)^{2}-\Omega^{2}}\right. \\
& \left.+\frac{k_{z}^{2}}{\left(\omega-n \Omega-k_{z} v_{z}\right)^{2}}\right]
\end{aligned}
$$


for a monoenergetic electron plasma [Fig. 1(b)]. As a check, this dispersion relation reduces to (19) in the limit $k_{z}=0$, and to (8) in the limit $k_{x}=0$.

For a general distribution function $f_{0}(\mathbf{v})$, we simply insert the integral $\int d^{3} \mathbf{v} f_{0}(\mathbf{v})$ to the right-hand member of Eq. (29), which now reads

$$
\begin{aligned}
k_{x}^{2}+k_{z}^{2}= & \omega_{p}^{2} \int d^{3} \mathbf{v} f_{0}(\mathbf{v}) \times \sum_{n=-\infty}^{\infty} J_{n}^{2}\left(\frac{k_{x} v_{\perp}}{\Omega}\right) \\
& \times\left[\frac{k_{x}^{2}}{\left(\omega-n \Omega-k_{z} v_{z}\right)^{2}-\Omega^{2}}+\frac{k_{z}^{2}}{\left(\omega-n \Omega-k_{z} v_{z}\right)^{2}}\right] .
\end{aligned}
$$

We shall show in Appendix B that Eq. (30) is identical to the Harris dispersion relation (23), even though they have very different appearances. Note that the infinite series (30) converges faster than that given in the original form, Eq. (23). All of the modes described in this paper are recovered from this dispersion relation, (23) or (30).

\section{CONCLUDING REMARKS}

In this paper, we provide an anatomy of velocity space instabilities without the tools of Vlasov equation. The entire calculation is done in the configuration space, for a single electron. The small signal displacement $\left(\mathbf{x}_{1}\right)$ of this electron immediately yields the macroscopic perturbation densities, correct to all order in its Larmor radius. The effect of velocity distribution is included only at the very end, almost as if it is an afterthought. The final result contains no more, and no less, information than the Vlasov approach, as exemplified by our reconstruction of the Harris dispersion relation. The direct dependence of the macroscopic space charge density on the displacement $\left(\mathbf{x}_{1}\right)$ [cf., Eq. (3)] provides a crucial link between space charge waves and velocity space instabilities, a problem of some confusion in the past. ${ }^{7}$

It is plain that this approach may be extended to electromagnetic modes and to the relativistic regime, yielding identical results as the Vlasov formulation, such as ${ }^{8}$ the cyclotron maser instability and the Wiebel instability. Other levels of approximation, such as the quasineutrality condition, may also be adopted once the macroscopic density given by Eq. (3) is computed. Kinetic modes involving magnetic perturbations can be constructed in an analogous manner.

This analysis may provide some simplications to the much studied collective modes excited by fusion-generated alpha particles, which are monoenergetic at birth. The present approach guarantees that all kinetic effects can be accounted for, and they can be tracked at each step because of the simplicity in the description in terms of the polarization variables.

Thus far, our attention has been mostly focussed on a uniform plasma. We argue here that this approach may also be applied to drift-like mode, in which there is a density gradient or temperature gradient in equilibrium. ${ }^{1}$ One needs only to calculate the displacement $\mathbf{x}_{1}$ of one electron (which has a typical set of constants of motion). The macroscopic density perturbation is still given by Eq. (3) which remains valid even if $n_{0}$ is a function of $x$. The effect of velocity distribution (representing electrons with different constants of motion) is inserted at the very end. Thus, all kinds of driftlike modes may be treated by an analysis very similar to the one given above, with all kinetic effects retained and without directly solving the Vlasov equation. Nonlocal effects ${ }^{1}$ may be identified at the outset, because of the conceptually much simpler formulation given here.

Flute-like modes ${ }^{1}$ can also be analyzed by the present approach, the modification being in the unperturbed orbit which must include the effect of magnetic field curvature. The rest of the calculations for $x_{1}$ and for $n_{1}$, and the integration over a velocity distribution (for different classes of particles), may proceed in a similar manner as done in this paper. Indeed, it is interesting to ponder on the relation between the treatment of kinetic modes that is given here, and the standard treatment of the hydromagnetic flute modes via the energy principle. Both treatments used the linearized displacement, $x_{1}$. In some sense, an energy principle has also been employed in the present study. ${ }^{6}$

Likewise, quasilinear theories may be developed along similar lines. In the absence of a phase space analysis, systematic expansions are likely to be more tractable. Nonlinear theory is more difficult to develop, however.

In summary, a simple procedure is demonstrated where velocity-space phenomena may be formulated mainly in the configuration space $(x, y, z)$, from the perturbed orbit of an electron (and ion).

\section{ACKNOWLEDGMENTS}

I wish to acknowledge the stimulating discussions with Ziya Akcasu, Ya. S. Derbenev, Ronald Gilgenbach, Bahman Hafizi, James Halloway, Terry Kammash, and Chuck Roberson.

This work was an outgrowth of my recent studies on several advanced vacuum electronic devices that require considerable attention to the space charge effects. As such, it was supported by the Naval Research Laboratory/Advanced Research Project Agency and by the Office of Naval Research.

\section{APPENDIX A: EQUIVALENCE BETWEEN EQ. (19) AND THE DISPERSION RELATION (20) FOR THE DORY-GUEST-HARRIS INSTABILITY}

To show that Eq. (19) is equivalent to Eq. (20), it suffices to prove

$$
\begin{aligned}
& \sum_{n=-\infty}^{\infty} \frac{J_{n}^{2}(b)}{(\omega-n \Omega)^{2}-\Omega^{2}} \\
& \quad=\frac{1}{\Omega^{2}} \sum_{n=-\infty}^{\infty}\left(\frac{n \Omega}{\omega-n \Omega}\right) \frac{1}{b} \frac{d}{d b} J_{n}^{2}(b) .
\end{aligned}
$$

The left-hand member of Eq. (A1) may be written as 


$$
\begin{gathered}
\sum_{n=-\infty}^{\infty}\left(\frac{1}{2 \Omega}\right) J_{n}^{2}(b)\left[\frac{1}{(\omega-n \Omega)-\Omega}-\frac{1}{(\omega-n \Omega)+\Omega}\right] \\
=\sum_{m=-\infty}^{\infty}\left(\frac{1}{2 \Omega}\right)\left[\frac{J_{m-1}^{2}(b)}{\omega-m \Omega}-\frac{J_{m+1}^{2}(b)}{\omega-m \Omega}\right]
\end{gathered}
$$

where, on the left-hand side of (A2), we have replaced $n+1$ by $m$ in the first term and $n-1$ by $m$ in the second term of the infinite sum. Upon using the Bessel function identities

$$
\begin{aligned}
& J_{m-1}(b)+J_{m+1}(b)=\frac{2 m}{b} J_{m}(b), \\
& J_{m-1}(b)-J_{m+1}(b)=2 \frac{d}{d b} J_{m}(b),
\end{aligned}
$$

in the right-hand member of (A2), we obtain the right-hand side of (A1). This completes the proof.

\section{APPENDIX B: EQUIVALENCE BETWEEN EQ. (30) AND THE HARRIS DISPERSION RELATION (23)}

To prove that Eq. (23) is equivalent to Eq. (30), we shall prove that the first term in the square bracket of Eq. (30) contributes exactly to the first term in the square bracket of Eq. (23), and that the second term in the squarc bracket of Eq. (30) contributes exactly to the second term in the square bracket of Eq. (23). We need to use the formula

$$
\int d^{3} \mathbf{v} f_{0}(\mathbf{v})=\int 2 \pi v_{\perp} d v_{\perp} \int d v_{z} f_{0}\left(v_{\perp}, v_{z}\right)
$$

We may represent the first term in the square bracket of Eq. (30) with the use of Eq. (A1), in which $\omega$ is replaced by $\omega-k_{z} v_{z}$, and $b$ by $k_{x} v_{\perp} / \Omega$. Performing integration by parts with respect to $v_{\perp}$ according to (B1), this first term of Eq. (30) immediately contributes to the first term in the square bracket of Eq. (23).

For the second term in the square bracket of Eq. (30), we perform integration by parts with respect to $v_{z}$ according to Eq. (B1). The result is simply the second term in the square bracket of (23). This manipulation has been used to obtain Eq. (10) from Eq. (9). This completes the proof.

${ }^{1}$ See, e.g., the following textbooks: F. F. Chen, Introduction to Plasma Physics and Controlled Fusion, second edition (Plenum, New York, 1984); K. Miyamoto, Plasma Physics for Nuclear Fusion, revised edition (MIT, Cambridge, MA, 1989); T. H. Stix, Waves in Plasmas (American Institute of Physics, New York, 1992).

${ }^{2}$ E. G. Harris, Phys. Rev. Lett. 2, 34 (1959); J. Nucl. Energy, Pt. C: Plasma Phys. 2, 138 (1961).

${ }^{3}$ R. A. Dory, G. E. Guest, and E. G. Harris, Phys. Rev. Lett. 14, 131 (1965).

${ }^{4}$ I. B. Bernstein, Phys. Rev. 109, 10 (1958).

${ }^{5}$ D. L. Bobroff, IRE Trans. Electron Devices ED-6, 68 (1959).

${ }^{6}$ This procedure is equivalent to calculating the work done on the radio frequency (RF) mode by the RF current carried by the electrons. It is implicit in the Vlasov formulation, but is ubiquitous in the literature in all $\mathrm{RF}$ sources, such as gyrotron, free electron laser, traveling wave tubes, etc. See, e.g., Ref. 7 for a recent review. In this regard, it is interesting to note here that the classic paper on Landau damping by J. M. Dawson, Phys. Fluids 4, 869 (1961), also drew upon the small signal power theorem that was formulated in the microwave tube community. The works cited in Ref. 8 below provide a sample of the treatments of the relativistic Vlasov equation.

${ }^{7}$ Y. Y. Lau and D. Chernin, Phys. Fluids B 4, 3473 (1992).

${ }^{8}$ K. K. Chu and J. L. Hirshfield, Phys. Fluids 21, 461 (1978); R. C. Davidson, Physics of Nonneutral Plasmas (Addison-Wesley, Redwood City, CA, 1990); High Power Microwaves, edited by V. L. Granatstein and I. Alexeff (Artech House, Norwood, MA, 1987). 